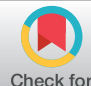

Check for
updates

\section{Case Report}

$\mathrm{J}$ Cerebrovasc Endovasc Neurosurg. 2020 June;22(2):85-89

Received: 12 June 2015

Revised: 27 March 2016

Accepted: 14 June 2016

\section{Correspondence to}

Kenneth C. Liu

Department of Neurosurgery, University of Virginia, P.O. Box 800212, Charlottesville, VA 22908, USA

Tel +1-434-924-2735

Fax +1-434-924-9656

E-mail kcl3j@hscmail.mcc.virginia.edu ORCID http://orcid.org/0000-0001-6217-1022

\footnotetext{
* This work was previously presented as a poster abstract at the American Association of Neurological Surgeons $84^{\text {th }}$ Annual Scientific Meeting, April 30-May 4, 2016; Chicago, IL, USA
}

\footnotetext{
This is an Open Access article distributed under the terms of the Creative Commons Attribution Non-Commercial License (http://creativecommons.org/licenses/ by-nc/3.0/) which permits unrestricted noncommercial use, distribution, and reproduction in any medium, provided the original work is properly cited.
}

\title{
Microvascular decompression of the posterior cerebral artery for treatment of oculomotor nerve palsy
}

\section{Jonathan Pomeraniec ${ }^{1}$, Dale Ding ${ }^{2}$, Alexander Ksendzovsky ${ }^{3}$, Kenneth C. Liu ${ }^{4}$}

${ }^{7}$ National Institute of Neurological Disorders and Stroke, Surgical Neurology Branch, National Institutes of Health, Bethesda, MD, USA

${ }^{2}$ Department of Neurological Surgery, University of Louisville School of Medicine, Louisville, KY, USA

${ }^{3}$ Department of Neurosurgery, University of Maryland School of Medicine, Baltimore, MD, USA

${ }^{4}$ Department of Neurological Surgery, University of Southern California, Los Angeles, CA, USA

Oculomotor nerve palsy resulting from non-aneurysmal vascular compression is extremely rare. Microvascular decompression (MVD) has been previously shown to improve oculomotor nerve palsy (ONP) secondary to arterial compression. A 71-year-old female, with a history of Cushing's disease previously treated with two transsphenoidal resections and Gamma Knife radiosurgery, presented with one year of progressive left eye diplopia and was diagnosed with a partial left oculomotor nerve palsy. We performed an orbitozygomatic craniotomy for MVD of the left posterior cerebral artery, which was found to be compressing the oculomotor nerve against the tentorium. Unfortunately, the patient's partial ONP remained unchanged at one year follow-up. The present case suggests inconsistent outcomes of MVD for ONP. Patients with prior sellar or parasellar irradiation may be less likely to benefit from this treatment approach.

Keywords Microsurgery, Microvascular decompression, Oculomotor nerve, Posterior cerebral artery, Skull base

\section{INTRODUCTION}

Oculomotor nerve palsy (ONP) secondary to non-aneurysmal vascular compression is extremely rare..$^{1-3) 9-11) 13) 14) 19)}$ Microvascular decompression (MVD) has been previously demonstrated to improve ONP secondary to arterial compression, although in a very limited number of cases. ${ }^{18)}$ The aim of this case report is to describe the technical aspects of MVD of the posterior cerebral artery (PCA) for the treatment of ONP. 


\section{CASE REPORT}

A 71-year-old female with a history of Cushing's disease, previously treated with two transsphenoidal resections and subsequent Gamma Knife radiosurgery (GKRS), presented with one year of progressive diplopia. Neuro-ophthalmologic examination revealed limitations in elevation, adduction, and depression as well as ptosis, anisocoria and lid retraction on attempted downgaze of her left eye. The patient was diagnosed with a partial left ONP. The possibility of a delayed cranial neuropathy from GKRS of pituitary adenoma was considered as a possible etiology of ONP. However, analysis of the GKRS treatment plan noted that the left lateral cavernous sinus wall was outside of the radiation field. Magnetic resonance imaging (MRI), 3D-sampling perfection with application optimized contrasts using different flip-angle evolution (SPACE) sequence, demonstrated compression of the left oculomotor nerve between the ipsilateral PCA and superior cerebellar artery (SCA) at the P1-P2 junction (Fig. 1A). In order to prevent further progression of her ONP and in an attempt to facilitate recovery, we elected to proceed with MVD of the left PCA.

We performed a left-sided modified orbitozygomatic craniotomy for an approach to the anterior cranial fossa. The Sylvian fissure was widely split from a distal to proximal approach. After proceeding down the subfrontal corridor, the optic nerve was identified and overlaying arachnoid was sharply opened and subsequently connected to the proximal Sylvian fissure. The opticocarotid cistern was opened and cerebrospinal fluid was drained to aid in brain relaxation. The carotid bifurcation was identified, as well as the proximal A1 and $\mathrm{M} 1$ segments. The temporal lobe was then retracted posteriorly to expose the tentorial incisura along with the inferior surface of the left supraclinoid internal carotid artery (ICA). The infundibulum of the left posterior communicating artery (PCOM) was identified, as well as the anteriorly displaced loop of the left PCA. This was lifted up, and the oculomotor nerve appeared to be significantly flattened by the pulsations of the left PCA against the tentorial edge (Fig. 1B). Further whitish discoloration of the nerve was seen at the distal edge of the compression. The left SCA was visualized below the oculomotor nerve, and the arachnoid bands surrounding this complex were cut and relaxed.

We explored the possibility of ligating the PCOM at its origin from the ICA in order to mobilize the PCA posteriorly. However, we noted too many thalamoperforators arising from the proximal segment of the PCOM to safely perform this maneuver. Instead, cottonoid buffers were placed between the left oculomotor nerve and PCA, which appeared to adequately decompress the nerve (Fig. 1C).

The patient had an uncomplicated postoperative course. Immediately after surgery, the patient had a complete left ONP, which subsequently improved, after a short course of steroids, to her baseline function by the time of discharge on postoperative day two. Postoperative 3D-SPACE MRI showed a small cerebrospinal fluid space between the left oculomotor nerve and the ipsilateral PCA and SCA (Fig. 1D). At one year neuro-ophthalmologic follow-up, the patient's oculomotor nerve function had not significantly improved from her preoperative baseline.

\section{DISCUSSION}

The present case suggest that the patient's ONP primarily resulted from vascular compression by the ipsilateral PCA, and that outcomes following MVD for the treatment of ONP may be more variable than previously recognized. There are few previously reported cases of ONP resulting from non-aneurysmal vascular compression. ${ }^{1-3) 9-1113) 14) 19)}$ Of these reports, only four cases involved compressive effects by the PCA (Table 1). ${ }^{1013) 14118)}$ Two of these cases described acute oculomotor paresis involving compression by the P1 segment that were not treated with surgical intervention; one was a postmortem diagnosis and the other involved a perimesencephalic vascular anomaly with partial ONP. ${ }^{1013)}$ Suzuki et al. reported a case of left oculomotor nerve palsy secondary to compression between an arteriosclerotic 

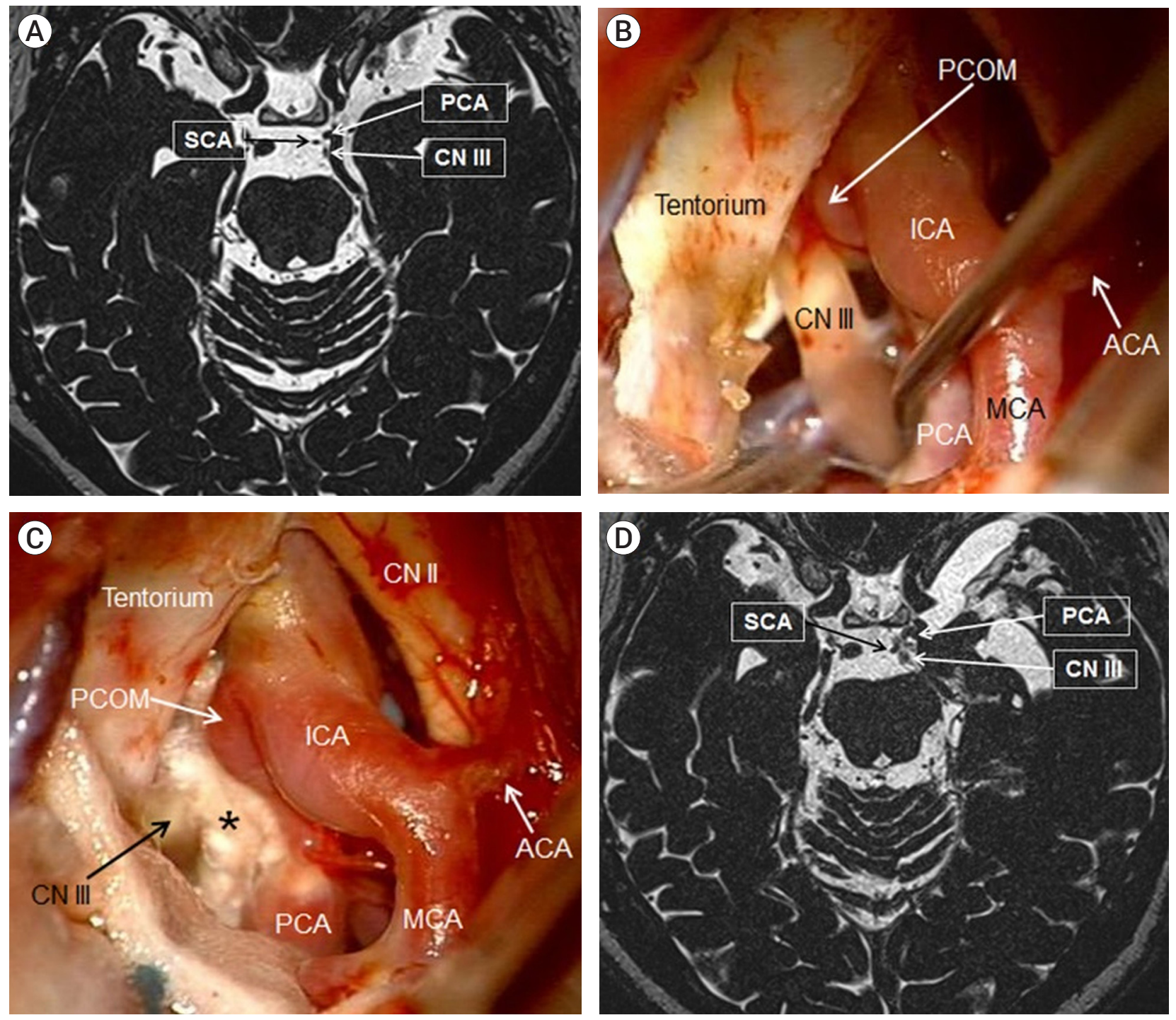

Fig. 1. (A) Preoperative brain MRI, axial 3D-sampling perfection with application optimized contrasts using different flip-angle evolution (SPACE) sequence, shows compression of the left CN III between the PCA and SCA at the P1-P2 junction. Beyond this point of compression, the nerve is decreased in volume (not shown). (B) Intraoperative photograph, from a transsylvian approach through a leftsided modified orbitozygomatic craniotomy, shows compression of CN III, which appears flattened, by the PCA (elevated by an instrument) against the tentorium. (C) Intraoperative photograph after placement of a cottonoid buffer (asterisk) between CN III and the PCA. (D) Postoperative MRI, axial 3D-SPACE sequence, shows a small cerebrospinal fluid space between CN III and the left PCA and SCA. MRI, magnetic resonance imaging; PCA, posterior cerebral artery; SCA, superior cerebellar artery, PCOM, posterior communicating artery; ICA, internal carotid artery; MCA, middle cerebral artery; ACA, anterior cerebral artery.

PCA and SCA. ${ }^{18)}$ A similar case was reported earlier by Nakagawa et al., in which a patient experienced relief with MVD of the left PCA but subsequently developed similar ONP on the contralateral side due to postsurgical arachnoid adhesions at the tentorial edge. ${ }^{14)}$ In both cases, ONP caused directly by arterial compression was more reliably detected intraoperatively than with preoperative MRI, and MVD was associated with nearcomplete resolution of the cranial neuropathy within three months. Non-aneurysmal arterial compression has also been reported in a case of an enlarged, atherosclerotic loop of the PCOM in a patient with unilateral subacute ONP and retro-orbital headaches in the setting of a small, non-distorting meningioma of the left tentorial 
Table 1. Summary of case reports of non-aneurysmal compression of the oculomotor nerve by the PCA

\begin{tabular}{|c|c|c|c|c|c|c|}
\hline Series (year) & $\begin{array}{l}\text { Patient age/ } \\
\text { gender }\end{array}$ & $\begin{array}{l}\text { Laterality and } \\
\text { degree of ONP }\end{array}$ & $\begin{array}{c}\text { Vascular anomaly } \\
\text { in addition to PCA } \\
\text { compression }\end{array}$ & Treatment & $\begin{array}{l}\text { Follow-up } \\
\text { duration }\end{array}$ & Outcome \\
\hline Current study & $71 / F$ & Left, partial & None & MVD (left) & 12 months & No improvement \\
\hline Suzuki et al. $(2008)^{18)}$ & $76 / M$ & Left, complete & None & MVD (left) & 1 month & Improved \\
\hline $\begin{array}{l}\text { Nakagawa et al. } \\
(1991)^{14)}\end{array}$ & $59 / M$ & $\begin{array}{l}\text { Bilateral (first on } \\
\text { left and then two } \\
\text { months later on } \\
\text { right) }\end{array}$ & $\begin{array}{l}\text { Duplicated } \\
\text { PcomA, P1 } \\
\text { infundibulum }\end{array}$ & $\begin{array}{l}\text { MVD (first on } \\
\quad \text { left and } \\
\text { then on right) }\end{array}$ & 3 months & $\begin{array}{l}\text { Improved ocular } \\
\text { movement and ptosis } \\
\text { at one month; improved } \\
\text { adduction at two months; } \\
\text { improved elevation at } \\
\text { three months }\end{array}$ \\
\hline $\begin{array}{l}\text { Morimoto et al. } \\
(1985)^{13)}\end{array}$ & $61 / F$ & Left, complete & None & $\begin{array}{l}\text { Postmortem } \\
\text { diagnosis }\end{array}$ & $\mathrm{N} / \mathrm{A}$ & $\begin{array}{l}\text { Patient developed acute } \\
\text { ONP after angiogram } \\
\text { complicated by left MCA } \\
\text { infarct and died one } \\
\text { month later }\end{array}$ \\
\hline Imes et al. $(1984)^{10)}$ & $31 / \mathrm{M}$ & Left, partial & $\begin{array}{c}\text { Small } \\
\text { perimesencephalic } \\
\text { vascular anomaly }\end{array}$ & $\begin{array}{l}\text { No surgical } \\
\text { intervention }\end{array}$ & $\mathrm{N} / \mathrm{A}$ & No improvement \\
\hline
\end{tabular}

ONP, oculomotor nerve palsy; PCA, posterior cerebral artery; MVD, microvascular decompression; PcomA, posterior communicating artery; MCA, middle cerebral artery

incisura. $^{2)}$

The natural history of ONP secondary to non-aneurysmal vascular compression is unknown. Previous studies of surgical outcomes of aneurysmal oculomotor nerve compression may serve as a proxy. ${ }^{2122}$ A review of 319 cases of isolated unilateral oculomotor palsy ( $70 \%$ complete, $30 \%$ partial) caused by intracranial aneurysms treated with surgical decompression proposed a correlation between treatment and symptomatic resolution: $64 \%$ of patients experienced complete recovery of oculomotor nerve function when surgery was performed within two weeks of symptom onset, $30 \%$ when surgery was performed within 14-30 days, and 14\% when surgery was performed after one month. Overall, $41 \%$ of patients experienced full resolution of symptoms. ${ }^{12)}$ Other studies have proposed that if patients experience complete oculomotor nerve recovery, then this will typically occur within three months of surgery. ${ }^{78)(15)}$

Our case describes a patient who underwent MVD nearly one year after the onset of ONP. At one year postoperative follow-up, the patient's oculomotor nerve function had not significantly improved from her preoperative baseline. This outcome suggests an inconsistent efficacy of MVD for ONP. While prior reports did not stratify the timing of postoperative recovery, their findings suggest that longer intervals between diagnosis and surgical management are associated with a lower likelihood of functional recovery. Delayed decompression in the present case may have contributed to lack of symptomatic improvement. Furthermore, the patient underwent prior GKRS for a pituitary adenoma, which may suggest that those who have undergone prior sellar or parasellar irradiation are less likely to benefit from MVD for the treatment of ONP. ${ }^{4-6) 16) 17)}$ Therefore, our case differs from prior reports of successful MVD for ONP in two aspects: (1) the relatively longer time interval between diagnosis and surgery, and (2) a history of prior sellar irradiation.

\section{CONCLUSIONS}

ONP is rarely caused by non-aneurysmal vascular compression. Previous reports suggest that clinical improvement can be achieved after treatment with MVD, particularly when performed early after symptom onset. The present case suggests inconsistent postoperative outcomes from MVD for ONP. Patients who have re- 
ceived prior sellar or parasellar irradiation may be less likely to benefit from surgical intervention. Until additional data from larger patient cohorts becomes available, the application of MVD should not be generalized to all cases of ONP secondary to non-aneurysmal vascular compression.

\section{Disclosure}

The authors report no conflict of interest concerning the materials or methods used in this study or the findings specified in this paper.

\section{REFERENCES}

1. Albayram S, Ozer H, Sarici A, Murphy K, Miller N. Unilateral mydriasis without ophthalmoplegia--a sign of neurovascular compression? Case report. Neurosurgery. 2006 Mar;58(3):E582-3; discussion E582-3.

2. Babbitz JD, Harsh GR 4th. Concomitant ectatic posterior communicating artery and tentorial meningioma as a source of oculomotor palsy: case report. Neurosurgery. 2005 Dec;57(6):E1316; discussion E1316.

3. D'Andrea F, Maiuri F, Gangemi M, Iaconetta G. Megadolichobasilar anomaly. Clinical and diagnostic considerations on 30 cases. Acta Neurol (Napoli). 1992 Aug-Dec;14(4-6):611-9.

4. Ding D, Starke RM, Kano H, Nakaji P, Barnett GH, Mathieu $\mathrm{D}$, et al. Gamma knife radiosurgery for cerebellopontine angle meningiomas: a multicenter study. Neurosurgery. 2014 Oct;75(4):398-408; quiz 408.

5. Ding D, Starke RM, Sheehan JP. Treatment paradigms for pituitary adenomas: defining the roles of radiosurgery and radiation therapy. J Neurooncol. 2014 May;117(3):445-57.

6. Ding D, Yen CP, Starke RM, Lee CC, Sheehan JP. Unyielding progress: recent advances in the treatment of central nervous system neoplasms with radiosurgery and radiation therapy. J Neurooncol. 2014 Sep;119(3):513-29.

7. Giombini S, Ferraresi S, Pluchino F. Reversal of oculomotor disorders after intracranial aneurysm surgery. Acta Neurochir
(Wien). 1991;112(1-2):19-24.

8. Hamer J. Prognosis of oculomotor palsy in patients with aneurysms of the posterior communicating artery. Acta Neurochir (Wien). 1982;66(3-4):173-85.

9. Hashimoto M, Ohtsuka K, Akiba H, Harada K. Vascular compression of the oculomotor nerve disclosed by thinslice magnetic resonance imaging. Am J Ophthalmol. 1998 Jun;125(6):881-2

10. Imes RK, Monteiro ML, Hoyt WF. Ophthalmoplegic migraine with proximal posterior cerebral artery vascular anomaly. J Clin Neuroophthalmol. 1984 Dec;4(4):221-3.

11. Kojo N, Lee S, Otsuru K, Takagi S, Shigemori M, Watanabe M. A case of ophthalmoplegic migraine with cerebral aneurysm. No Shinkei Geka. 1988;16(5 Suppl):503-7.

12. Leivo S, Hernesniemi J, Luukkonen M, Vapalahti M. Early surgery improves the cure of aneurysm-induced oculomotor palsy. Surg Neurol. 1996 May;45(5):430-4.

13. Morimoto K, Nagatani M, Mogami H. A case simulating ophthalmoplegic migraine: clinicopathological study. Headache. 1985 Nov;25(8):426-8.

14. Nakagawa H, Nakajima S, Nakajima Y, Furuta Y, Nishi O, Nishi K. Bilateral oculomotor nerve palsies due to posterior cerebral arterial compression relieved by microvascular decompression--case report. Neurol Med Chir (Tokyo). 1991 Jan;31(1):45-8.

15. Peerless SJ, Hernesniemi JA, Gutman FB, Drake CG. Early surgery for ruptured vertebrobasilar aneurysms. J Neurosurg. 1994 Apr;80(4):643-9.

16. Starke R, Kano H, Ding D, Nakaji P, Barnett GH, Mathieu D, et al. Stereotactic radiosurgery of petroclival meningiomas: a multicenter study. J Neurooncol. 2014 Aug;119(1):169-76.

17. Starke RM, Przybylowski CJ, Sugoto M, Fezeu F, Awad AJ, Ding D, et al. Gamma Knife radiosurgery of large skull base meningiomas. J Neurosurg. 2015 Feb;122(2):363-72.

18. Suzuki K, Muroi A, Kujiraoka Y, Takano S, Matsumura A. Oculomotor palsy treated by microvascular decompression. Surg Neurol. 2008 Aug;70(2):210-2.

19. Zingale A, Chiaramonte I, Mancuso P, Consoli V, Albanese V. Craniofacial pain and incomplete oculomotor palsy associated with ipsilateral primitive trigeminal artery. Case report. J Neurosurg Sci. 1993 Dec;37(4):251-5. 